s990000 xx9999 SC english S 199835714715

\title{
Lattice disorder in strongly correlated lanthanide and actinide intermetallics
}

\author{
C. H. Boоth,${ }^{a *}$ E. D. Bauer, ${ }^{b}$ M. B. Maple,${ }^{b}$ J. M. Lawrence,${ }^{c}$ G. \\ H. KWEI ${ }^{d}$ AND J. L. SARRAO ${ }^{e}$ \\ ${ }^{a}$ Chemical Sciences Division, The Glenn T. Seaborg Center, Lawrence Berkeley \\ National Laboratory, Berkeley, California 94720, USA, ${ }^{b}$ Department of Physics and \\ the Institute for Pure and Applied Physics, University of California, San Diego, \\ California 92093, USA, ${ }^{c}$ Physics Department, University of California, Irvine, \\ California 92697, USA, ${ }^{d}$ Lawrence Livermore National Laboratory, Livermore, \\ California 94550, USA, and ${ }^{e}$ Los Alamos National Laboratory, Los Alamos, New \\ Mexico 87545, USA. E-mail: chbooth@lbl.gov \\ (Received 0 XXXXXXX 0000; accepted 0 XXXXXXX 0000)
}

\begin{abstract}
Lanthanide and actinide intermetallic compounds display a wide range of correlatedelectron behavior, including ferromagnetism, antiferromagnetism, nonmagnetic (Kondo) ground states, and so-called "non-Fermi liquid" (NFL) behavior. The interaction between $f$ electrons and the conduction band is a dominant factor in determining the ground state of a given system. However, lattice disorder can create a distribution of interactions, generating unusual physical properties. These properties may include NFL behavior in many materials. In addition, lattice disorder can cause deviations from standard Kondo behavior that is less severe than NFL behavior. We will review the lattice disorder mechanism within a tight-binding model and present measurements of the $\mathrm{YbBCu}_{4}$ and $\mathrm{UPd}_{x} \mathrm{Cu}_{5-x}$ systems demonstrating the applicability of


the model. These measurements indicate that while the ${\mathrm{Yb} B C \mathrm{u}_{4}}_{4}$ system appears to be well ordered, both site interchange and continuous bond-length disorder occur in the $\mathrm{UPd}_{x} \mathrm{Cu}_{5-x}$ series. Nevertheless, the measured bond-length disorder in $\mathrm{UPdCu}_{4}$ does not appear to be enough to explain the NFL properties simply with this Kondo disorder model.

\section{Introduction}

Many heavy-fermion $f$-electron compounds exhibit magnetic and electronic properties which do not behave as expected from the Landau Fermi-liquid theory thought to be applicable to heavy-fermion systems. When these anomalies include a certain subset of behavior, the materials are said to be non-Fermi liquids (NFL) (for a review, see Coleman et al., 1996). These behaviors include logarithmic or weak power law dependences of the Sommerfeld specific heat coefficient $\gamma(T)=C(T) / T$ and the magnetic susceptibility $\chi(T)$, and a deviation from $T^{2}$ of the temperature dependence of the electrical resistivity as $T \rightarrow 0 \mathrm{~K}$ (typically linear). Most but not all NFL materials are known to be crystallographically or chemically disordered, but theoretical treatments differ on whether this disorder is important to the NFL behavior (Millis, 1993, Bernal et al., 1995).

One focus of research has been to find NFL behavior in well-ordered materials. However, such materials are by definition stoichiometric, and therefore the singleimpurity Anderson model (SIAM) should no longer apply at some level. Unfortunately, the periodic Anderson model (PAM) that should apply to $f$-ions on a periodic, wellordered lattice, has not been solved, although progress is being made (Tahvildar-Zadeh et al., 1997). In any case, the search for well-ordered NFL materials must necessarily include attempts to isolate coherence effects as would be described by the PAM from those effects that will require a truly new description of the ground state, such as 
quantum critical behavior (Millis, 1993).

Lattice disorder plays a pivotal role both in attempts to understand coherence effects in Anderson lattice systems and in the search for NFL mechanisms and materials. In Anderson lattice systems, lattice disorder is a potential mask of real coherence effects because it can create a distribution of behavior in a material that may be difficult to assign to the lattice disorder. In NFL studies, disorder can cause NFL behavior (Bernal et al., 1995, Miranda al., 1997) and therefore can also be viewed as a mask to discovering well-ordered NFL materials. Moreover, the role of disorder in generating NFL behavior is still an open question. Kondo disorder models, whereby a distribution of Kondo temperatures ( $T_{K}$ 's) extends to sufficiently low temperatures, have been shown to be capable of generating NFL behavior without including any essentially new physical mechanisms. Recently, Castro-Neto et al. (1998) have proposed that the presence of sufficient disorder (lattice or otherwise) can drive a system into a Griffiths' phase, creating a distribution of quantum critical points. A Griffiths' phase has the potential of generating more universal behavior than the Kondo disorder model, and so may be a better candidate for NFL behavior in materials that are known to possess some lattice disorder.

Searching for lattice disorder can be a much more complicated and self-deceiving task than many researchers outside of crystallography realize. A common procedure in characterizing any material is to perform a powder diffraction measurement and to perform a Rietveld refinement of a proposed crystal structure at room temperature. If any lattice disorder is indicated by enhanced displacement parameters (the $u^{2}$ 's), it is often ignored in this procedure because only a temperature dependent measurement is capable of discerning between a large $u^{2}$ that is caused by significant weight in the phonon spectrum at low frequencies (i.e. a low Debye temperature for a given site) and one that is caused by static (i.e. non-phonon) disorder due to, for instance, off- 
center displacements or site-interchange between two atomic species within a given compound. Even if temperature dependent diffraction data are collected, it can be difficult to identify the source of disorder if it is particularly pathological, as in a case where site-interchange occurs but insufficient contrast exists between the scattering factors for the two species of atoms (for example, see Chau et al., 1998, for the case of $\left.\mathrm{UPdCu}_{4}\right)$.

X-ray-absorption spectroscopy can be an excellent tool for identifying certain kinds of disorder when used in conjunction with diffraction results. Perhaps the most unique feature is the ability to uniquely probe the local atomic arrangement around a given species of atom with the x-ray-absorption fine-structure (XAFS) technique. With this measurement, one can distinguish between a given atomic species that is sitting on two distinct sites in a structure as long as those two sites have significant differences in their local atomic arrangement.

The remainder of this paper begins with a discussion of how disorder can affect magnetic susceptibility. Experimental XAFS data will be presented for two systems: the Anderson lattice $\mathrm{YbBCu}$ system and the $\mathrm{NFL} \mathrm{UPd}_{x} \mathrm{Cu}_{5-x}$ system. The possibility of $B / \mathrm{Cu}$ site interchange will be explored, and temperature dependent displacement parameters will be carefully considered to search for any lattice disorder. Many of the details of the experimental analysis and results are described elsewhere (Booth et al., 1998, Lawrence et al., 2000, Bauer et al., 2001).

\section{Lattice disorder in the single impurity model}

A hallmark of the SIAM is the pronounced maximum in the magnetic susceptibility that occurs for $J>1 / 2$ as the Kondo singlet is formed at low temperatures. As one decreases temperatures from well above $T_{K}, \chi(T)$ increases following a typical CurieWeiss form. This increase slows down near $T_{K}$ and at approximately one-third of 
$T_{K}, \chi(T)$ begins to decrease, going to a constant at zero temperature that is inversely proportional to $T_{K}$. The relative size of the maximum in $\chi(T)$ to the zero-temperature constant $\chi(0)$ is only a function of the total angular moment $J$ on the $f$ ion, with the maximum $\chi(T)$ decreasing with decreasing $J$ (Rajan, 1983).

Another way to decrease the relative size of the maximum is to include lattice disorder. Such disorder will produce a distribution $P\left(T_{K}\right)$ of Kondo temperatures. The susceptibility can be calculated with $P\left(T_{K}\right)$ and a Bethe-ansatz calculation of the susceptibility of a single Kondo impurity, $\chi\left(T, T_{K}\right)$ (Rajan, 1983):

$$
\chi(T)=\int \chi\left(T, T_{K}\right) P\left(T_{K}\right) \mathrm{d} T_{K}
$$

$P\left(T_{K}\right)$ is calculated by starting with the expession for $T_{K}$ :

$$
T_{K}=E_{F} e^{-1 /(\rho \mathcal{J})}
$$

where $E_{F}$ is the Fermi energy, $\rho$ is the density of states at the Fermi level and $\mathcal{J}$ is the conduction-electron/local-moment exchange energy. The coupling energy $V_{\text {tot }}$ is related to the exchange energy in Kondo theory simply by $\mathcal{J}=V_{\text {tot }}^{2} / \epsilon_{f}$, where $\epsilon_{f}$ is the $f$-level energy. At this stage, we have described the generic Kondo disorder model put forth by Bernal et al. (1995). We now choose lattice disorder as the microscopic origin of the Kondo disorder. In order to include the lattice,we use a tight-binding approximation to obtain the contribution to the coupling energy of each pair of atoms $A$ ( $f$ ion) and $B$ (assuming $d$ electrons in the conduction band):

$$
V_{A, B}=\frac{\eta_{f d} \hbar^{2}}{m_{e}} \frac{\left(r_{\mathrm{A} f}^{5} r_{\mathrm{B} d}^{3}\right)^{1 / 2}}{R_{\mathrm{A}-\mathrm{B}}^{6}},
$$

where $r_{X \ell}$ is the radius of the electronic shell with angular momentum $\ell$ for atom $X$ (tabulated by Straub \& Harrison, 1985), and $R$ is the bond length between the atoms with $\ell=f$ and $d$. The coefficient $\eta_{f d}$ depends only on the $\ell$ 's and the bond symmetry (see Appendix B in Harrison \& Straub, 1987). We will assume all bonds are $\sigma$-bonds. 
The total hybridization energy is then $V_{\text {tot }}=\sum_{A, B \text { pairs }} V_{A, B}$. Within this tight-binding approach, calculated energies are often too high compared to experiment by a factor of two (Harrison \& Straub, 1987). However, we are only interested in relative trends because we choose other parameters (such as $E_{F}$ and $\rho$ ) to account for this absolute error.

With this model as a frame work, the connection between neighboring species is made through $r_{B}$, and through the actual bond length with $R_{A-B}$. Therefore, bondlength disorder will create a distribution of $V_{A, B}$ 's by replacing $R_{A-B}$ with a distribution of bond lengths, and site interchange will create a distribution of $V_{A, B}$ 's by varying the $r_{B}$ 's. An important difference between these two types of disorder is that bond-length disorder is defined to be continuous in this model (we will use a Gaussian distribution) and site-interchange is defined to be discrete: if one considers only the nearest neighbors in a calculation, there are only a finite number of possible combinations of the various $V_{A, B}$ terms.

Before we can demonstrate this model, we need to pick a system for which we can perform the sum over $V_{A, B}$. Consider the case of the $C 15 b$ structure that is formed by some compounds of the form $A B \mathrm{Cu}_{4}$. All atoms sit on the cubic $\overline{4} 3 m f c c$ lattice, with $A$ atoms sitting on the $4 a$ site at $(0,0,0), B$ atoms on the $4 c$ sites at $(0.25,0.25,0.25)$ and $\mathrm{Cu}$ atoms on $16 e$ sites forming a corner-shared tetrahedral network centered at $(0.75,0.75,0.75)$. Band structure calculations on the $C 15 b$ material $\mathrm{YbAgCu}_{4}$ indicate that the $\mathrm{Cu}$ and $\mathrm{Ag} d$ electrons make the main contributions to the conduction band (Monachesi and Continenza, 1996). Therefore, in the calculations that follow, we will sum over the $12 A$-Cu nearest neighbors at $\sim 2.93 \AA$ and over the 4 A-B next nearest neighbors at $\sim 3.06 \AA$, as shown in Fig. 1(a). We also assume that $E_{\mathrm{F}}$ and $\rho$ are fixed within a given compound and do not have their own distributions.

Figure 2 shows how the addition of (continuous) bond-length disorder can affect the 
susceptibility within this Kondo disorder model. The effect on the actual width of the distribution is pronounced, with only $\sim 0.01 \AA$ of extra bond-length disorder necessary to produce a width of the $P\left(T_{K}\right)$ of $\sim 40 \%$. Surprisingly, this apparently large width of $P\left(T_{K}\right)$ has little effect on the calculated $\chi(T)$. In fact, lattice disorder continues to have little effect on $\chi(T)$ until one reaches a Debye-Waller width of about $\sigma \sim 0.04 \AA$. At this point, a significant amount of weight in $\mathrm{P}\left(T_{K}\right)$ exists at lower temperatures. A previous study of the Kondo disorder model (Bernal et al., 1995) has shown that if enough weight exists near $0 \mathrm{~K}$, logarithmic divergences in the magnetic susceptibility and the heat capacity are generated. Moreover, it has also been shown (Miranda et $a l ., 1997)$ that a linear temperature dependence to the resistivity is also generated when enough low- $T_{K}$ weight exists in $P\left(T_{K}\right)$. Therefore, one should appreciate how little intrinsic bond-length disorder is necessary to produce such a situation. Although such a distortion would be difficult to attain by applying external pressure, it is quite common to see changes in local bond lengths of this magnitude with chemical doping.

Another mechanism for creating a distribution of Kondo temperatures is site interchange, whereby two atomic species that nominally sit on distinct sites have a propensity to exchange sites. The ideas presented here are easily applied to off-stoichiometric samples where, for instance, in $\mathrm{UPd}_{0.5} \mathrm{Cu}_{4.5}$ one can expect to find copper atoms on $4 c$ sites, even in the nominal structure. The case we consider now is when in $A B \mathrm{Cu}_{4}$, some percentage of the $B$ atoms interchange sites with copper atoms. In this case, a distribution of possible local environments around the $f$-ion sites is created. Consider the nominally ordered case shown in Fig. 1(a) with $A$ as uranium and $B$ as $\mathrm{Pd}$, as in $\mathrm{UPdCu}_{4}$. If each $4 c$ site has a $25 \%$ probability of being $\mathrm{Cu}$ rather than $\mathrm{Pd}$, then each $16 e$ site as a $25 / 4=6.25 \%$ chance of being $\mathrm{Pd}$ rather than $\mathrm{Cu}$ (four times as many $16 e$ sites). With these probabilities, a binomial distribution of possible environments can be calculated. Using values of $E_{\mathrm{F}}$ and $\rho / \epsilon_{f}$ from Booth et al. (1998), we can calculate 
a discrete distribution of Kondo temperatures, as shown in Fig. 3. As should be immediately appreciated, there is roughly equal weight for Kondo temperatures ranging from $\sim 100 \mathrm{~K}$ to $250 \mathrm{~K}$ with this degree of site interchange.

A material that site interchanges can be thought of as a material that is chemically "self-doped" in that ions with different atomic radii now sit on nominally the same sites. Such site interchange can also create a distribution of bond lengths, as discussed above. For instance, the atomic radius of $\mathrm{Cu}$ is $\sim 1.57 \AA$, while Pd's atomic radius is $\sim 1.79 \AA$. Therefore, local distortions of $0.2 \AA$ may exist in the local environment around $\mathrm{U}$ atoms. Consequently, if site interchange exists, some level of bond-length disorder likely also exists. We have calculated the effect of bond-length disorder on the distribution of $T_{K}$ 's and display the results in Fig. 3(b). The calculations show that the discrete nature of the underlying distribution is quickly washed out by a bond-length disorder of only about $0.01 \AA$. This result attests to the overwhelming sensitivity of this model to bond-length disorder. Again, significant weight is not generated in the low $T_{K}$ region until about $0.04 \AA$ of disorder is included.

To summarize the results of this section, both continuous and discrete distributions of Kondo temperatures can be generated with various types of disorder. Truly discrete distributions seem unlikely, since even when the conditions necessary to create a discrete distribution exist, namely some kind of chemical substitution occurs, these substitutions will also generate some bond-length disorder, which will quickly overwhelm the discrete character of any $P\left(T_{K}\right)$. However, the width of a distribution created by chemical substitution can readily be quite large, thus enhancing the overall width when bond-length disorder is included. 


\section{Experimental examples}

\subsection{Experimental details}

XAFS experiments were performed at the $B$ edges for $B=\mathrm{Tl}, \mathrm{In}, \mathrm{Cd}$ and $\mathrm{Ag}$ in samples of $\mathrm{YbBCu}_{4}$ and at the $\mathrm{U} L_{\mathrm{III}}, \mathrm{Pd} K$ and $\mathrm{Cu} K$ edges for members of the $\mathrm{UPd}_{x} \mathrm{Cu}_{5-x}$ series. Data were collected on BL 4-3 at the Stanford Synchrotron Radiation Laboratory (SSRL) using a half-tuned $\mathrm{Si}(220)$ double crystal monochromator. Samples were ground, typically passed through a $30 \mu \mathrm{m}$ sieve and brushed onto scotch tape to obtain a uniform thickness. Strips of tape were stacked to obtain an absorption jump at the various edges of less than unity. The $\mathrm{YbBCu}$ samples are the same fluxgrown crystals described in Sarrao et al. (1999). Sample preparation of the $\operatorname{UPd}_{x} \mathrm{Cu}_{5-x}$ samples is consistent with that described in Chau et al. (1998), and will be described elsewhere (Lawrence et al., 2001). Samples with $1.0 \leq x \leq 1.5$ show NFL behavior both in resistivity and magnetic susceptibility measurements.

\subsection{Kondo coherence and $\mathrm{YbBCu_{4 }}$}

In a recent paper, the properties of many members of the $\mathrm{YbBCu}_{4}$ series were compared (Sarrao et al., 1999). Kondo temperatures range from $\sim 60 \mathrm{~K}$ for $\mathrm{YbZnCu}_{4}$ to $\sim 750 \mathrm{~K}$ for $\mathrm{YbTlCu}_{4}$. Although many properties of these materials can be ex-

plained with the SIAM, $\chi_{\max } / \chi(0)$ was significantly less than predicted by the SIAM for $\mathrm{YbZnCu}_{4}, \mathrm{YbCdCu}_{4}$ and $\mathrm{YbMgCu}_{4}$. The possibility that crystal fields were responsible for this disagreement has been ruled out by recent inelastic neutron scattering measurements (Lawrence et al., 2000). As is clear from Fig. 2, the reduction in $\chi_{\max } / \chi(0)$ could very easily be due to lattice disorder. If this final possibility is ruled out, then coherence effects can be seriously taken as a possible explanation, opening the door for comparisons to PAM theories.

Since the $\mathrm{Yb} L_{\mathrm{III}}$ and $\mathrm{Cu} K$ edges nearly overlap, we restrict this study to the XAFS 
from the $B$ atoms. We searched for two kinds of disorder: $B / \mathrm{Cu}$ site interchange, as indicated by the presence of a short $B$-Cu bond (see Fig. 1(b)), and any other disorder by measuring the temperature dependence of the $B$-Cu and $B$-Yb Debye-Waller factors and comparing the measurements to the correlated-Debye model (Crozier et al., 1988).

The XAFS technique is very well suited for searching for $B / \mathrm{Cu}$ site interchange in these compounds because the nominal local environment around the $B(4 c)$ sites is very different than around the $\mathrm{Cu}$ (16e sites). As shown in Fig. 1, the nearest-neighbors around the $4 c(B)$ sites are 12 copper atoms at $\sim 2.9 \AA$, followed by $4 \mathrm{Yb}$ atoms at $\sim 3.06 \AA$. This environment differs from the $16 e$ sites, which have 6 copper neighbors at $\sim 2.5 \AA$. Our task is therefore reduced to looking for a peak in the XAFS spectrum corresponding to a $B$-Cu neighbor at $\sim 2.5 \AA$, which is $\sim 0.4 \AA$ shorter than in the nominal structure.

XAFS data were reduced using standard methods described elsewhere. The data are presented and fit in $r$-space. Figure 4 shows data for $\mathrm{YbCdCu}_{4}$ and $\mathrm{YbAgCu}_{4}$, using only scattering paths corresponding to the nominal structure, that is, site interchange was not included. The fit quality is very high. When some $B / \mathrm{Cu}$ site interchange is included in the fit, the fit quality does not improve significantly, and we place an upper limit of $5 \%$ of the $B$ atoms possibly sitting on $16 e$ sites.

With site interchange removed as a potential source of disorder, we choose the nominal crystal structure for our fitting model. Data were collected at various temperatures between $20 \mathrm{~K}$ and $300 \mathrm{~K}$. The variance of the $B$ - $\mathrm{Cu}$ and the $B$-Yb bond-length distribution are shown in Fig. 5. The variance results were then fit to the following equation:

$$
\sigma_{\text {fit }}^{2}(T)=\sigma_{\text {stat }}^{2}+F\left(T, \Theta_{\mathrm{cD}}\right)
$$

where $\sigma_{\text {stat }}^{2}$ is a temperature independent offset due to positional disorder and $F\left(T, \Theta_{\mathrm{cD}}\right)$ 
is given by the correlated-Debye model, with $\Theta_{\mathrm{cD}}$ as the correlated-Debye temperature. The fits shown in Fig. 5 use a $\Theta_{\mathrm{cD}} \approx 250 \mathrm{~K}$ for all pairs, and a maximum level of $\sigma_{\text {stat }}^{2}$ of $<0.001 \AA^{2}$ was obtained. The estimated error on this measurement of $\sigma_{\text {stat }}^{2}$ is roughly $0.0005 \AA^{2}$, so all of these measurements are generally consistent with no lattice disorder.

\subsection{Disorder models and NFL behavior in $U P d_{x} C u_{5-x}$}

Our previous study (Booth et al., 1998) of $\mathrm{UPdCu}_{4}$ found that $\sim 25 \%$ of $\mathrm{Pd}$ atoms sit on nominally Cu sites. As shown in Figs. 2 and 3, this level of site interchange will have a large effect on the measured magnetic susceptibility. Therefore, the disorder must be accounted for in any theory of the magnetic susceptibility in these materials. By including $\sim 0.05 \AA$ of additional bond-length disorder, the magnetic susceptibility could be reasonably reproduced by the model in Sec. 2. The addition of this extra disorder was necessary to obtain enough weight in the $P\left(T_{K}\right)$ distribution near $0 \mathrm{~K}$ to generate the NFL behavior. However, it is also possible to create a collection of $0 \mathrm{~K}$ quantum critical points with sufficient disorder. Such a phase is called a Griffiths' phase. Within the Griffiths' phase model, a range in phase space is created that might be called a "critical line" at $0 \mathrm{~K}$. In $\mathrm{UPd}_{x} \mathrm{Cu}_{5-x}$ this line would extend from $x \approx 1.0$ to above 1.5. At the present time, it is difficult to directly compare the Kondo disorder and Griffiths' phase models because there is no Griffiths' phase calculation starting from a measured amount of lattice disorder and arriving at a magnetic susceptibility. In any case, we expect that less disorder is necessary in a Griffiths' phase model than in a Kondo disorder model. It is therefore very important to test whether the assumed addition of $\sim 0.05 \AA$ of bond-length disorder can be verified by experiment.

Such a verification requires a study of many members of the $\mathrm{UPd}_{x} \mathrm{Cu}_{5-x}$ series in order to better determine certain factors such as the site interchange and amplitude 
reduction factors ( $S_{0}^{2}$ 's). We have performed XAFS measurements of samples with various $x$ 's, and have successfully isolated the Debye-Waller factors for the U-Cu nearest-neighbor peaks. These fits are complex because the site interchange must be known (as measured from the Pd edge data) and then applied to the U edge fits so that the number of $\mathrm{U}-\mathrm{Pd}(16 e)$ pairs underneath the main $\mathrm{U}-\mathrm{Cu}(16 e)$ peak can be properly accounted for. In each case, the $\mathrm{Cu}$ edge fits were used as a cross check. These data were collected as a function of temperature, and the same analysis as described in Sec. 3.2. was applied. The results are summarized in Fig. 6 .

Interestingly, although some residual static disorder is measured (especially for $x=1$ ), the measured level of disorder is less than that required for this Kondo disorder model for all $x$. Further details will be given in a future article (Bauer et al., 2001).

\section{Discussion and Conclusions}

We have tried to demonstrate the significance of lattice disorder in $f$-electron systems, both as a potential source of "uncharacteristic" behavior in the temperature dependence of materials that otherwise obey a single impurity model, and in systems where the low temperature properties are not well described by any model, namely the nonFermi liquids. The problem of identifying gross disorder and applying knowledge of this disorder is relatively straight forward when the tight-binding approximation is employed, but it is nevertheless important to recognize certain distinctions. For instance, although a small amount of lattice disorder can produce a very wide distribution of $T_{K}$ 's, such a distribution can still produce a magnetic susceptibility consistent with no disorder. However, if enough disorder exists such that weight at low $T_{K}$ 's develops, large effects in the magnetic susceptibility can be expected, possibly even NFL behavior. 
Applying these concepts to the $\mathrm{YbBCu}$ series, we find that lattice disorder is likely not the cause of deviations from the SIAM. We now believe that no other explanation exists for these deviations other than collective effects that are only possible in a lattice of $f$-ions, that is, some form of the PAM is necessary to describe the deviations from the SIAM. We note that other behavior is consistent with a slower-than-expected crossover from the low temperature Fermi liquid state to the high temperature local moment state (Lawrence et al., 2001). This result likely corresponds to the "protracted screening" recently predicted for the Anderson lattice (Tahvildar-Zadeh et al., 1997).

Our previous measurements of $\mathrm{UPdCu}_{4}$ demonstrated the importance of accounting for $\mathrm{Pd} / \mathrm{Cu}$ site interchange in understanding the magnetic and electronic properties. However, a closer look at the U-Cu bond-length disorder indicates that there does not appear to be enough for a simple Kondo disorder model to apply, opening the door to other theories that include disorder, such as the Griffiths' phase.

Work at Lawrence Berkeley National Laboratory was supported by the Office of Basic Energy Sciences (OBES), Chemical Sciences Division of the Department of Energy (DOE), Contract No. DE-AC03-76SF00098. We acknowledge the support of the National Science Foundation, DMR-97-05454. Experiments were performed at SSRL, which is operated by the DOE/OBES.

\section{References}

Bauer, E. D., Booth, C. H., Maple, M. B., and Kwei, G. H. (2001). In preparation.

Bernal, O. O., MacLaughlin, D. E., Lukefahr, H. G., Andraka, B. (1995). Phys. Rev. Lett. 75, 2023-2023.

Booth, C. H., MacLaughlin, D. E., Heffner, R. H., Chau, R., Maple, M. B., and Kwei, G. H. (1998). Phys. Rev. Lett. 81 3960-3963.

Castro-Neto, A. H., Castilla, G, and Jones, B. A. (1998). Phys. Rev. Lett. 81,3531-3534.

Chau, R., Maple, M. B., and Robinson, R. A. (1998). Phys. Rev. B 58, 139-143. 
Coleman, P., Maple, M. B., and Millis, A. J. (ed) (1996). Institute for Theoretical Physics Conf. on Non-Fermi Liquid Behavior in Metals (Santa Barbara, CA, 1996), J. Phys.: Condens. Matter 8.

Crozier, E. D., Rehr, J. J., and Ingalls, R. (1988). X-ray Absorption: Principles, Applications, Techniques of EXAFS, SEXAFS, XANES, edited by D. Konigsberger and R. Prins, pp. 373-442. New York: Wiley.

Harrison, W. A. and Straub, G. K. (1987). Phys. Rev. B 36, 2695-2706.

Lawrence, J. M., Riseborough, P. S., Booth, C. H., Sarrao, J. L., Thompson, J. D., and Osborn, R. (2001). Phys. Rev. B, in press. A preprint can be found in cond-mat/0006147.

Monachesi, P, and Continenza, A. (1996). Phys. Rev. B 54, 13558-13565.

Millis, A. J. (1993). Phys. Rev. B 48, 7183-7196.

Miranda, E., Dobrosavlijevi(c), and Kotliar, G. (1997). Phys. Rev. Lett. 78, 290-293.

Rajan, V. T. (1983). Phys. Rev. Lett. 51, 308-311.

Sarrao, J. L., Immer, C. D., Fisk, Z., Booth, C. H., Figueroa, E., Lawrence, J. M., Modler, R., Cornelius, A. L., Hundley, M. F., Kwei, G. H., Thompson, J. D., and Bridges, F. (1999). Phys. Rev. B 59, 6855-6866.

Straub, G. K. and Harrison, W. A. (1985). Phys. Rev. B 31, 7668-7679.

Tahvildar-Zadeh, A. N., Jarrell, M., Freericks, J. K. (1997). Phys. Rev. B 55, R3332-3335. 

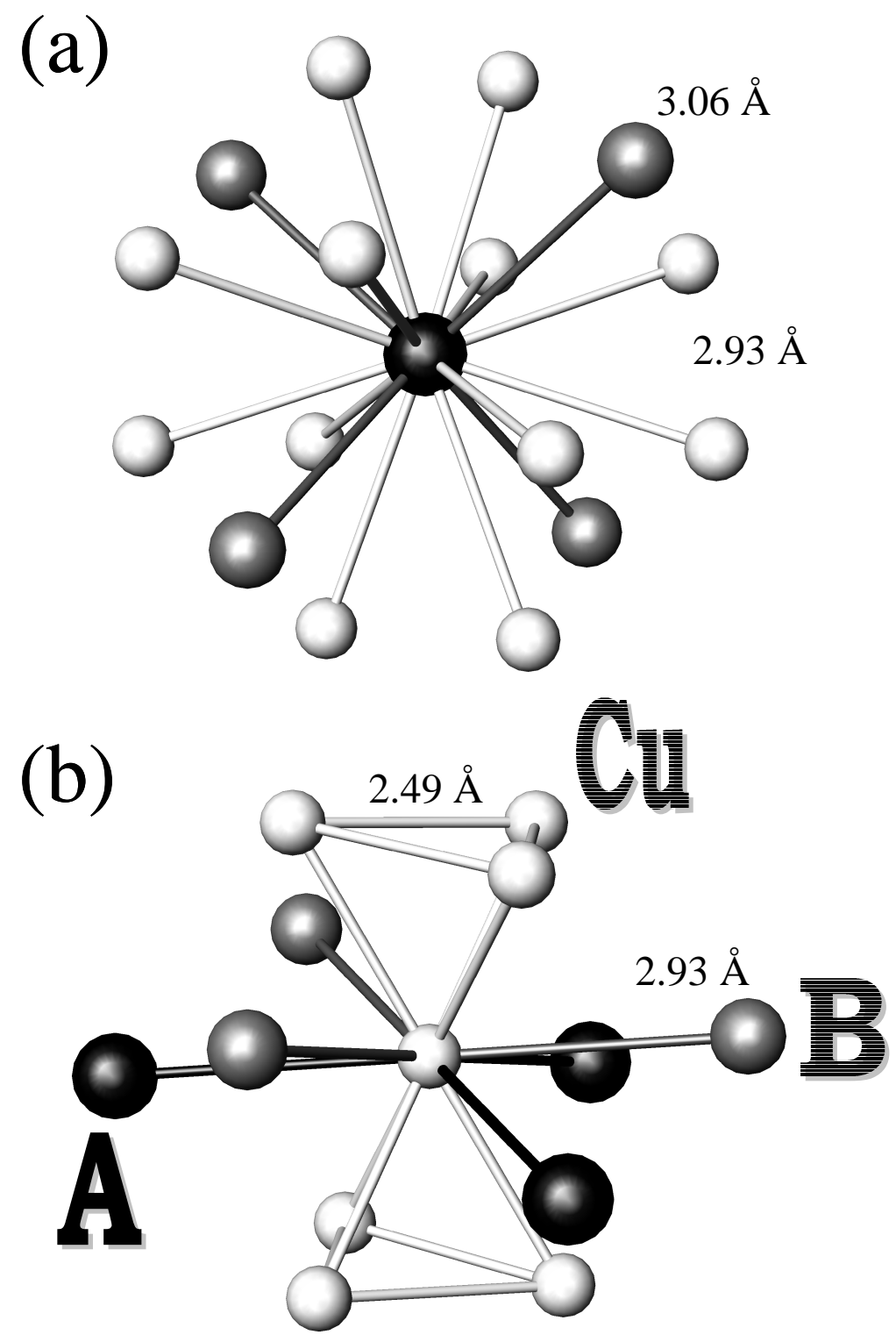

Fig. 1. The local structure in the $C 15 b$ crystal structure. Panel (a) shows the local structure around the $f$-ion (atom $A$ ), but also represents the environment around atom $B$ by switching all $A$ and $B$ atoms in the panel. Panel (b) shows the local structure around the $\mathrm{Cu}$ atoms. Bond lengths are for $\mathrm{YbAgCu}_{4}$. 

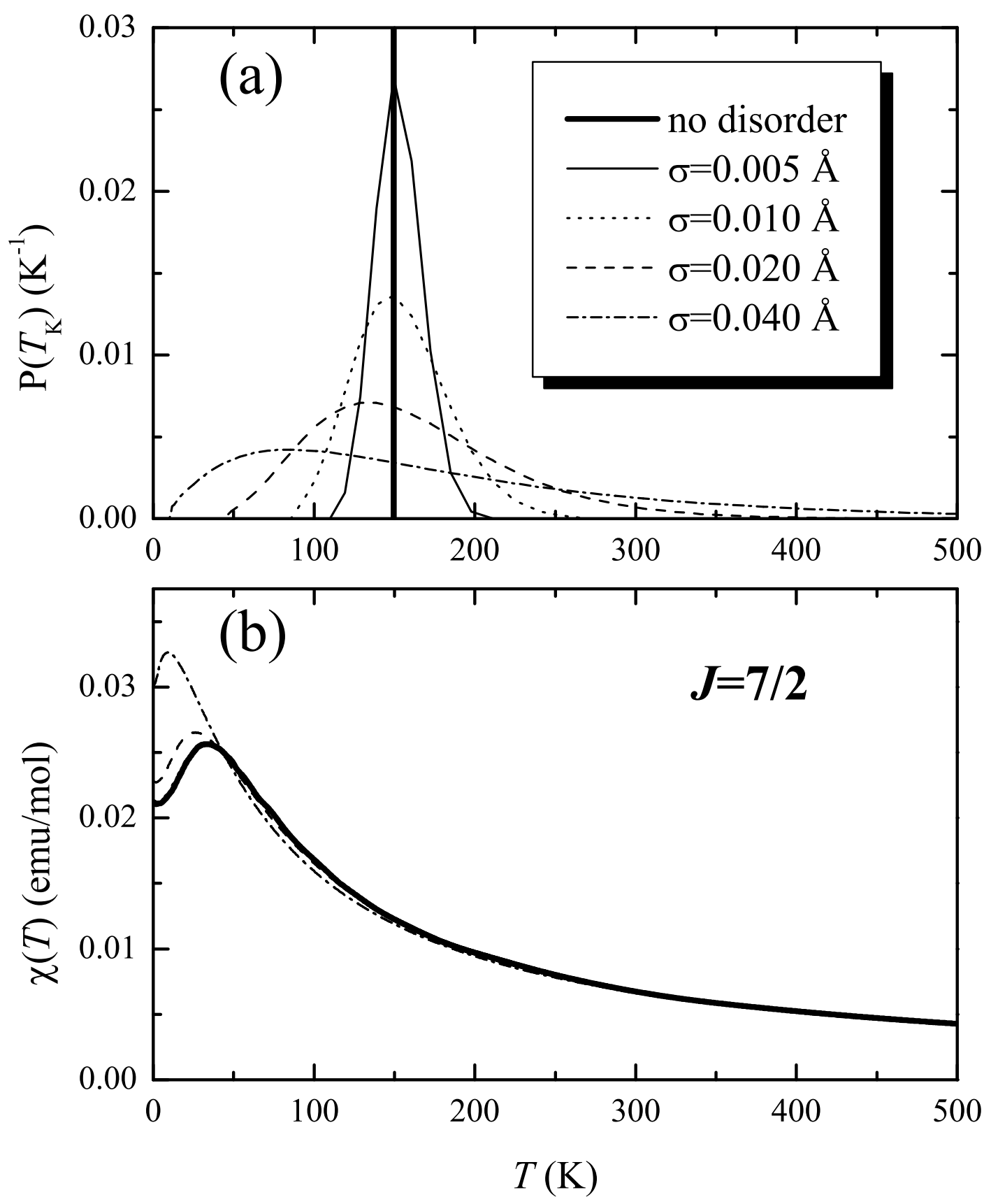

Fig. 2. (a) The calculated distribution of Kondo temperatures assuming a Gaussian distribution of near-neighbor bond lengths in the $C 15 b$ crystal structure of $\mathrm{YbAgCu}_{4}$. Other input parameters such as atomic species, $E_{\mathrm{F}}$, and $\rho / \epsilon_{f}$ were chosen to give $T_{K}=150 \mathrm{~K}$. (b) The calculated $\chi(T)$ using the distributions in panel (a). 

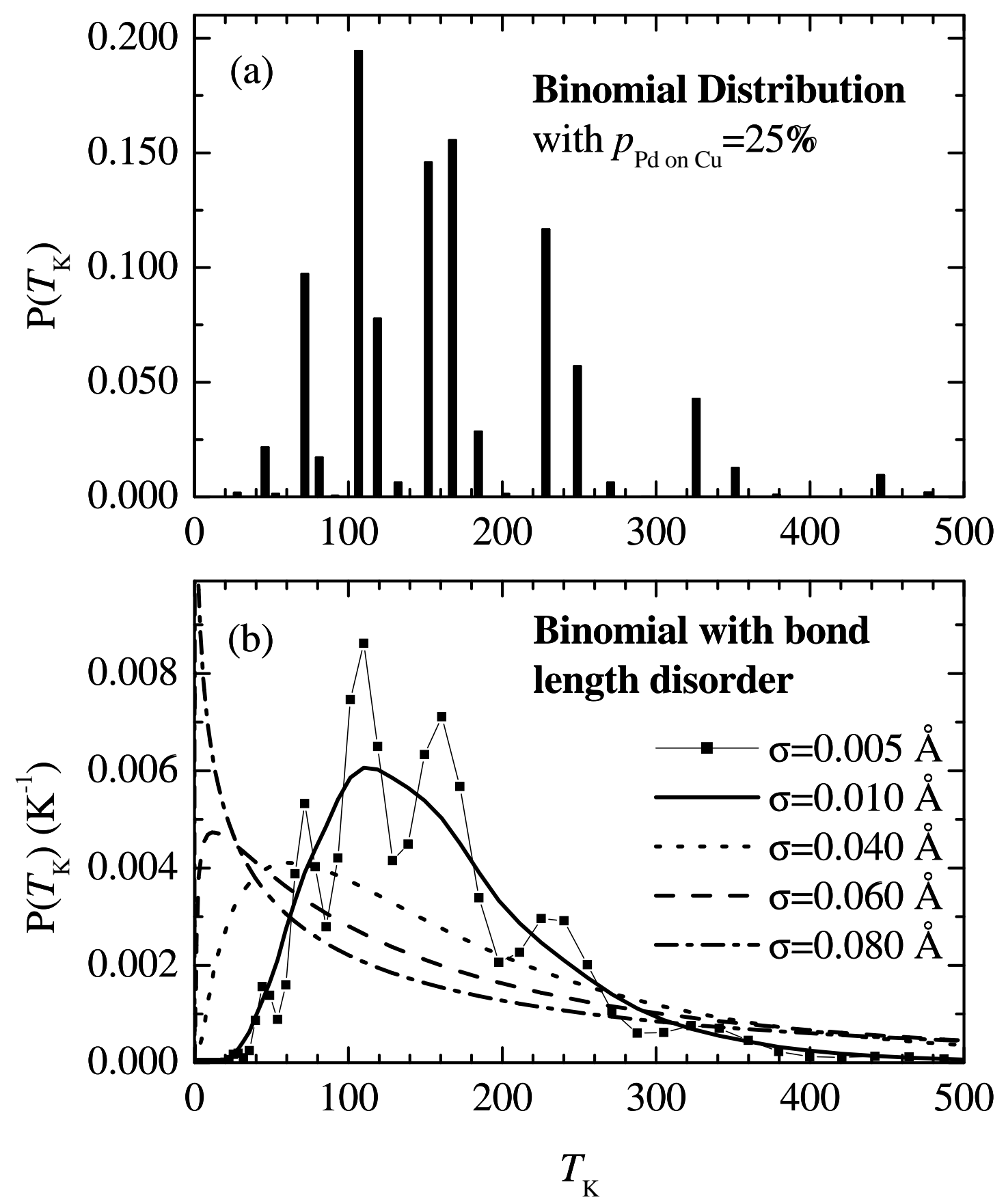

Fig. 3. (a) The discrete distribution of $T_{K}$ 's based on $25 \% \mathrm{Pd} / \mathrm{Cu}$ site interchange in $\mathrm{UPdCu}_{4}$. (b) The same distribution as in panel (a) convolved with the indicated amount of bond-length disorder. 


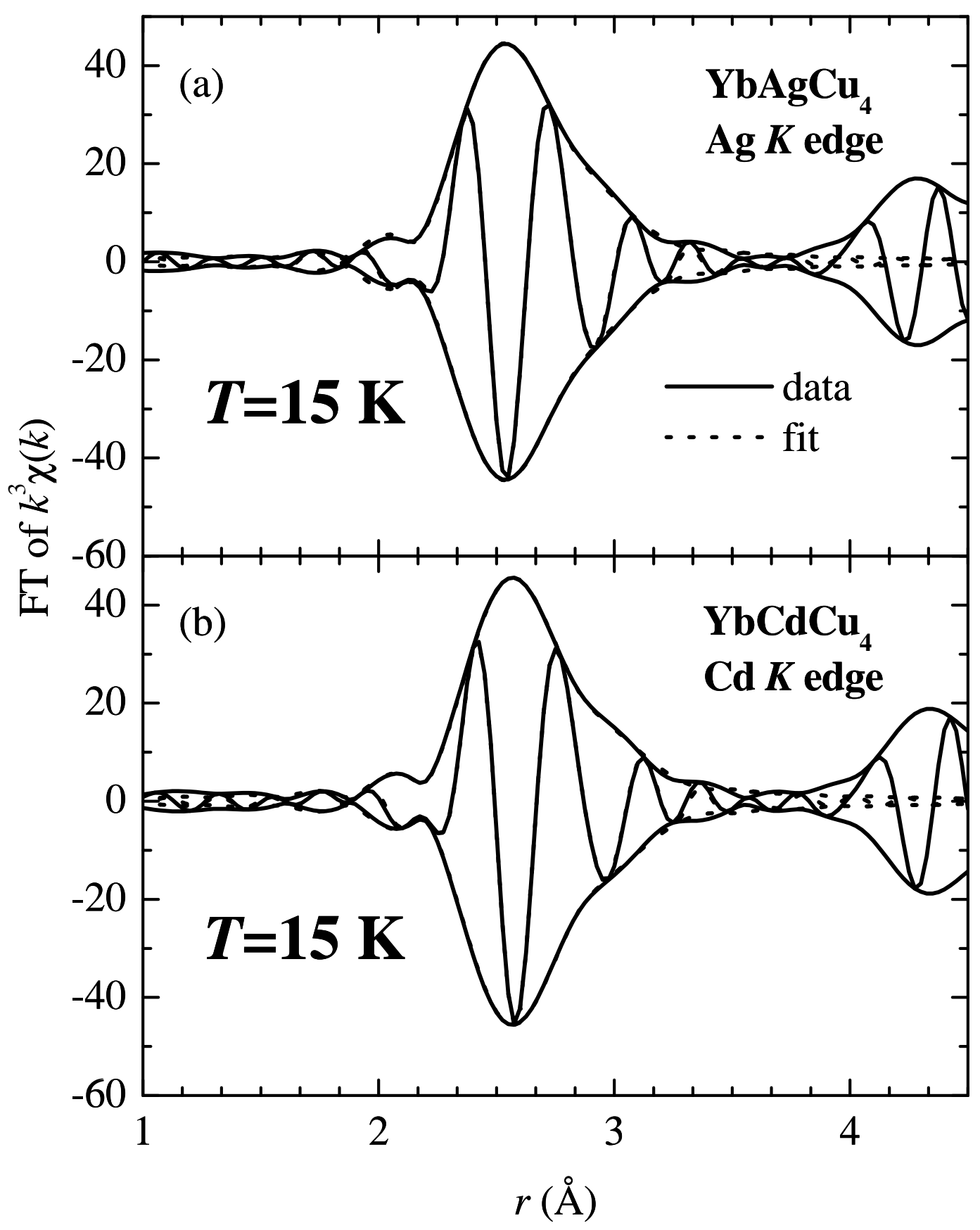

Fig. 4. The Fourier transform (FT) of $k^{3} \chi(k)$ for (a) $\mathrm{YbAgCu}_{4}$ and (b) $\mathrm{YbCdCu}_{4}$. The outer envelope represents \pm the amplitude and the oscillatory line within the envelope is the real part of the complex transform. These transforms are from 2.5-15 $\AA^{-1}$ and Gaussian narrowed by $0.3 \AA^{-1}$. Fits are from 2.0 to $3.2 \AA$. 

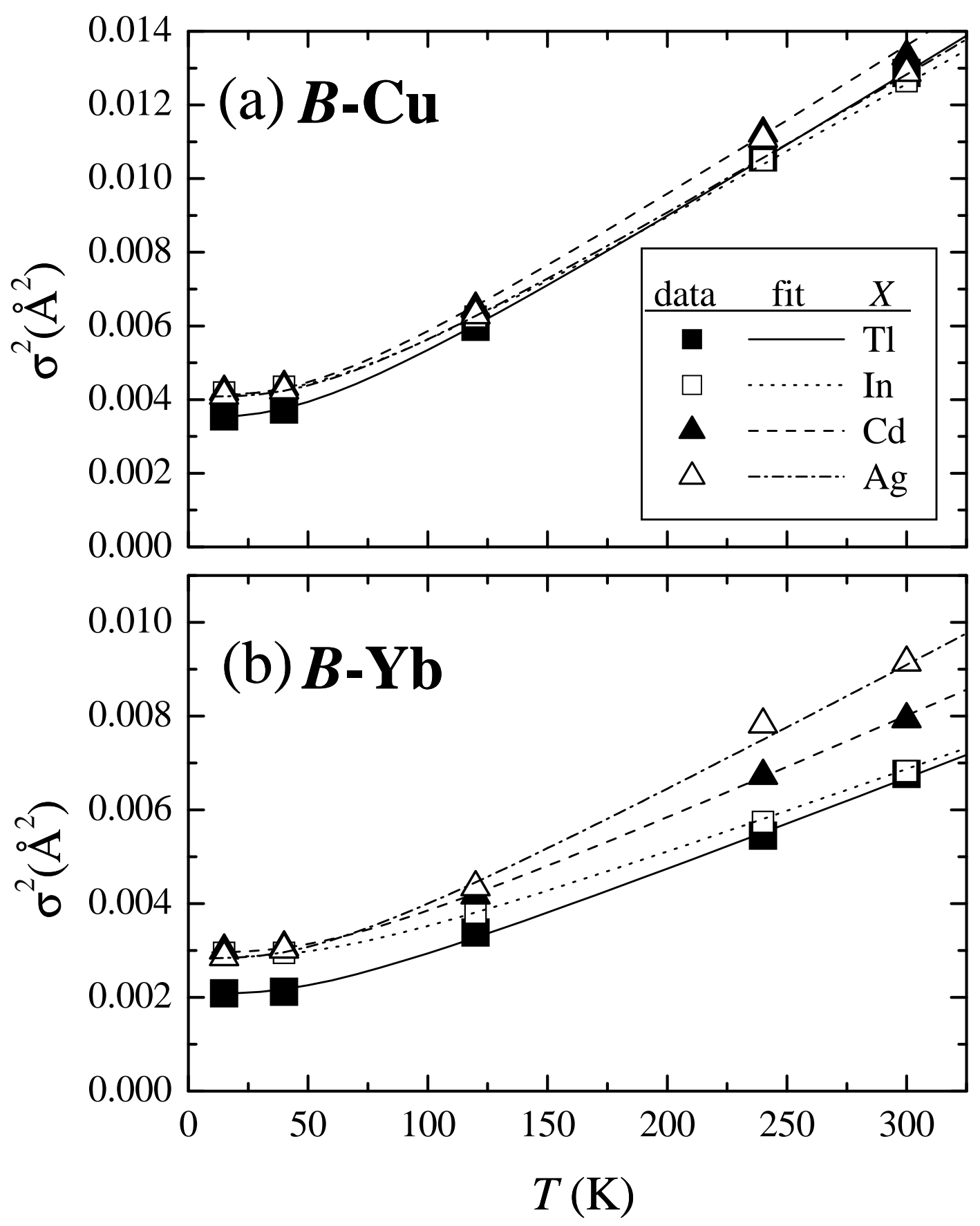

Fig. 5. The measured variance $\left(\sigma^{2}\right)$ of the bond-length distribution for the $B$-Cu pairs at $\sim 2.93 \AA$ and the $B$-Yb pairs at $\sim 3.06 \AA$ for $B=\mathrm{Tl}, \mathrm{In}, \mathrm{Cd}$ and $\mathrm{Ag}$. The fits are to a correlated-Debye model, as described in the text. 


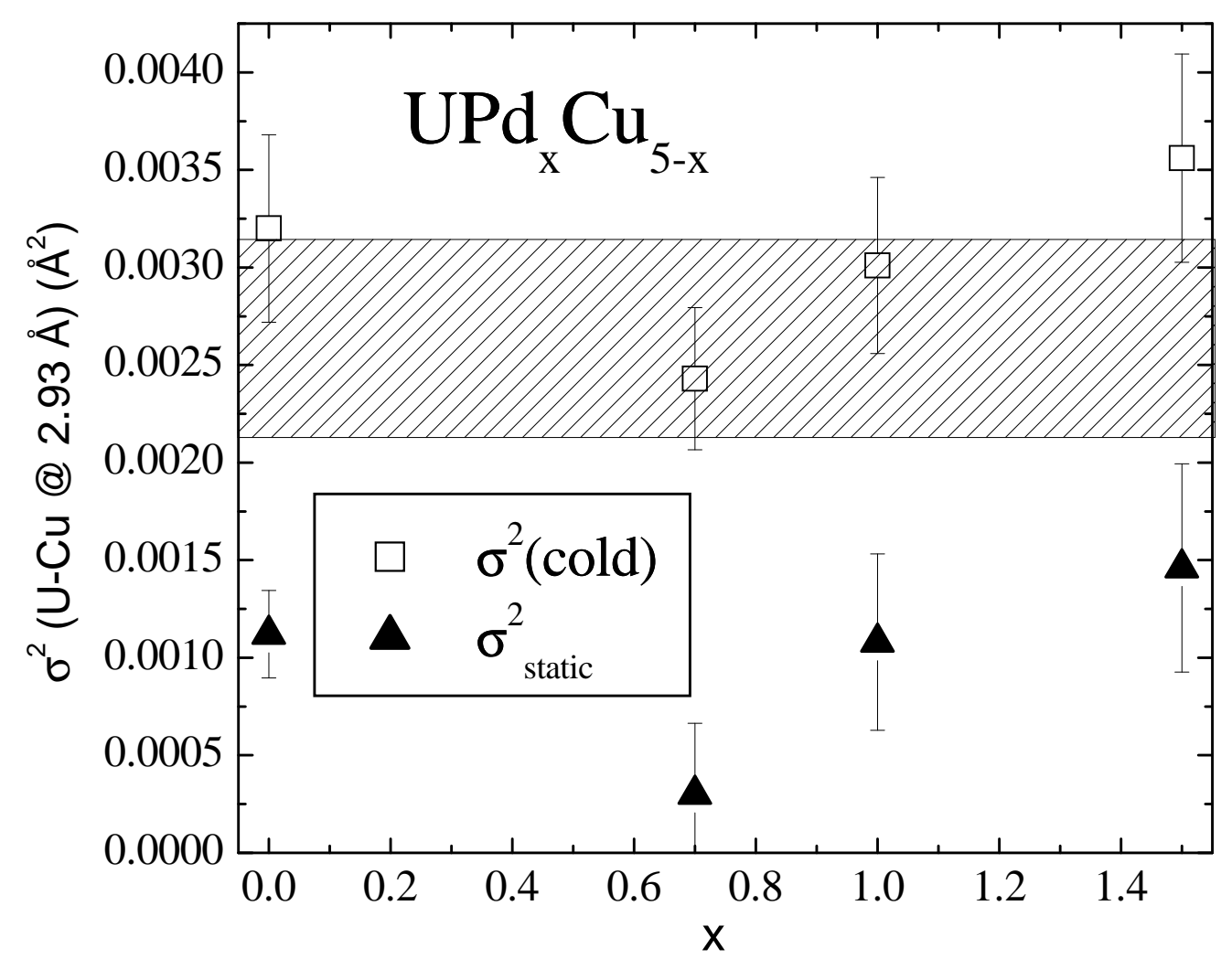

Fig. 6. The bond-length distribution variance for the U-Cu pairs at $\sim 2.93 \AA$ as a function of $x$ in $\mathrm{UPd}_{x} \mathrm{Cu}_{5-x}$. The "cold" results are for the lowest temperature collected for that value of $x$, between $4-20 \mathrm{~K}$. $\sigma_{\text {static }}^{2}$ was determined by fits of the temperature dependent data to the correlated-Debye model, as described in the text. The hashed area shows the approximate amount of disorder necessary for lattice disorder to fully explain NFL behavior in the $x=1.0$ and 1.5 compounds based on the Kondo disorder model. 